\title{
Nimodipine-loaded mixed micelles: formulation, compatibility, pharmacokinetics, and vascular irritability study
}

This article was published in the following Dove Press journal:

International Journal of Nanomedicine

12 July 2012

Number of times this article has been viewed

\author{
Xu Songl,* \\ Yu Jiang ${ }^{2, *}$ \\ Chunjuan Ren' \\ Xun Sun' \\ Qiang Zhang ${ }^{3}$ \\ Tao Gong' \\ Zhirong Zhang' \\ 'Key Laboratory of Drug Targeting \\ and Drug Delivery System, Ministry \\ of Education, West China School \\ of Pharmacy, Sichuan University, \\ Chengdu, ${ }^{2}$ Center for Drug \\ Evaluation, State Food and Drug \\ Administration, ${ }^{3}$ State Key Laboratory \\ of Natural and Biomimetic Drugs, \\ School of Pharmaceutical Sciences, \\ Peking University, Peking, People's \\ Republic of China \\ *These authors contributed equally \\ to this work
}

Background: The clinical application of nimodipine (NIM) is limited by several unfavorable properties, which are induced by its low aqueous solubility. In the present study, nimodipineloaded egg phosphatidylcholine-sodium glycocholate mixed micelles (NIM-EPC-SGC-MMs) were prepared to improve the water solubility of NIM, thus allowing it to be more applicable for clinical use.

Methods: NIM-EPC-SGC-MMs were prepared using the coprecipitation method and the factors influencing formulation quality were optimized. After formulation, water solubility, solubilizing efficiency, drug loading, particle size, physical compatibility, pharmacokinetics, and vascular irritability were determined.

Results: The mean size of the NIM-EPC-SGC-MMs was $6.099 \pm 0.048 \mathrm{~nm}$ under optimized conditions. The water solubility of NIM in EPC-SGC-MMs was enhanced 250-fold compared with free NIM. The physical compatibility, pharmacokinetic, and vascular irritability studies showed that, in comparison to the commercially available NIM injections, NIM-EPC-SGCMMs presented better physical compatibility, the same pharmacokinetic profile, and less risk of local vascular irritation and phlebitis.

Conclusion: EPC-SGC-MMs represent a promising new formulation suitable for the intravenous delivery of NIM.

Keywords: drug loading, nimodipine injection, physical compatibility, solubilizing efficiency

\section{Introduction}

Nimodipine (NIM) is a 1,4-dihydropyridine calcium channel blocker (Figure 1), which has been shown to dilate cerebral arterioles and increase cerebral blood flow. ${ }^{1}$ It has been reported to possess beneficial pharmacological activity in the treatment of a range of cerebrovascular disorders. ${ }^{1,2}$ The therapeutic use of NIM includes that in subarachnoid hemorrhage, focal or global ischemia, and epilepsy. ${ }^{2,3}$ Presently, NIM is also used for acute ischemic stroke, migraines, cerebral vasospasm, multi-infarct dementia, and hypotension-induced memory impairment. ${ }^{4-9}$ Despite the promising biological effects of NIM, its clinical performance is restricted by its low oral bioavailability (as low as $10 \%)$ and low aqueous solubility $(3.86 \mu \mathrm{g} / \mathrm{mL}) .{ }^{9,10}$ Thus, intravenous administration is the alternative route for NIM in case of efficacy. To achieve a sufficient NIM concentration, a commercial injection of NIM has been produced by solubilizing NIM with about $40 \%$ solvent mixture: $23.7 \%(\mathrm{v} / \mathrm{v})$ ethanol and $17 \%(\mathrm{v} / \mathrm{v})$ polyethylene glycol $400 .{ }^{11}$ However, this concentration has many noticeable drawbacks when applied in the clinic. For instance, according to the intravenous dosage regimen, this formulation
Correspondence: Tao Gong; Zhirong Zhang Key Laboratory of Drug Targeting and Drug Delivery System, Ministry of Education, Sichuan University, No 17, Section 3, Southern Renmin Road, Chengdu 61004I, People's Republic of China Tel +862885501615 Email gongtaoy@126.com; zrzzl@vip.sina.com 


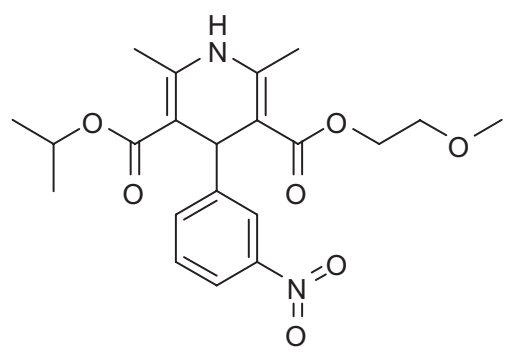

Figure I Chemical structure of nimodipine.

requires a time-consuming administration (about 10 hours by infusion pump), as well as special equipment and additional nursing care. ${ }^{11,12}$ Further, NIM-ethanol injections may cause local adverse reactions at the administration site, such as pain and inflammation. ${ }^{9,11}$ Of more concern, there may be phlebitis during infusion and poor patient compliance. In addition, crystallization caused by the poor water solubility of NIM can also occur when the NIM injection is diluted by injection solutions, which is dangerous to patients. ${ }^{13}$ Consequently, the application of these NIM commercial injections has been limited by their high medication cost, low patient compliance, and safety considerations.

Thus, there is a pressing need to develop a safe, injectable formulation for NIM by means of pharmaceutics. Based on its physiological compatibility and solubilizing capacity, a phosphatidylcholine (PC)-bile salts (BS)-mixed micelle (MM) formulation is an attractive candidate for the intravenous administration of poorly soluble drugs. ${ }^{14-20}$ This simple approach of combining a water-soluble surfactant with a water-insoluble phospholipid results in an isotropically clear solution. ${ }^{21,22}$ Many studies have been published regarding the preparation and characterization of PC-BS-MMs. ${ }^{16,23-26}$ BS present in bile with a high concentration can solubilize $\mathrm{PC}$ to a large extent, forming a kind of clear mixed-micellar solution that in turn can solubilize substances with poor water solubility. ${ }^{16,19}$ With the solubilized phospholipids, the hemolytic effects of BS can be neutralized. ${ }^{27}$ Most importantly, PC-BS-MMs have been proven to be locally and systemically well tolerated, with no embryotoxic, teratogenic, or mutagenic effects after administration. ${ }^{28}$ Without a toxicologically solvent mixture, a PC-BS-MM formulation can reduce irritation and improve the patient compliance. Consequently, PC-BS-MMs are an ideal drug carrier for the intravenous administration of NIM.

In this study, our objective was to develop a novel potential delivery vehicle for NIM that could be used in the clinic. First, we prepared and characterized NIM-loaded egg phosphatidylcholine (EPC)-sodium glycocholate
(SGC)-mixed micelles (MMs). We then compared and studied the pharmacokinetic properties of NIM-EPC-SGCMMs versus NIM injection. We also compared the physical compatibility of the infusion solutions and the intravenous irritation of NIM-EPC-SGC-MMs compared with NIM injection.

\section{Materials and methods Reagents and chemicals}

NIM was kindly supplied by Chongqing Yaoyou Pharmaceutical Co, Ltd (Chongqing, China). EPC (purified ovolecithin), purity $80 \%$, was obtained from Lipoid $\mathrm{GmbH}$ (Ludwigshafen, Germany). Pyrene was purchased from Sigma Aldrich (Munich, Germany) and used without further purification. SGC was a gift from Chongqing Yaoyou Pharmaceutical Co, Ltd. The commercially available NIM injection $(0.2 \mathrm{mg} / \mathrm{mL}$, batch number: 110101$)$ was purchased from Ya Bao Pharmaceutical Group Co, Ltd (Shanxi, China). Reference substances of nitrendipine (number: 100585-200602) and NIM (number: 100270-200002) were purchased from Chengdu Rong Jin Medical Technology Development Co, Ltd (Chengdu, China). Sodium chloride injection (batch number: 1191022) was purchased from Taiji Group Southwest Pharmaceutical Co, Ltd (Chongqing, China). Doubledistilled (DI) water and the other chemical reagents were of analytical grade or better.

\section{Animals}

Male Wistar rats (weight $200 \pm 20$ g) and Japanese big-ear rabbits (weight $2.5-3.0 \mathrm{~kg}$ ) were provided by the Laboratory Animal Center of Sichuan University (Chengdu, China). The protocols and procedures were recognized by the Institutional Animal Care and Use Committee of Sichuan University.

\section{Preparation of NIM-EPC-SGC-MMs}

Due to the good solubility of NIM, SGC, and EPC in anhydrous ethanol, NIM was easily loaded into PC-BS-MMs using the coprecipitation method. ${ }^{26}$ Briefly, NIM, SGC, and EPC (at different molar ratios and concentrations) were dissolved by anhydrous ethanol in a $25 \mathrm{~mL}$ round-bottom flask. The organic solvent was evaporated at $60^{\circ} \mathrm{C}$ under vacuum to form a thin film that was then rehydrated in physiological saline and dispersed by slight shaking before the $\mathrm{pH}$ was adjusted to about 7.4. To remove the unloaded drug, the solution was centrifuged at $10,000 \times g$ in an Allegra X-22R Centrifuge with a F2402H rotor (Beckman Coulter Inc, Brea, CA) for 5 minutes and the supernatant containing freshly formed MMs was collected. 
Based on the single-factor method, a high-quality formulation was obtained by optimizing several main formulation factors, including the ratio of the mass concentration of EPC and SGC, the total concentration of EPC and SGC, the dose of NIM, and the $\mathrm{pH}$ value of dispersed medium. Each experiment was run in triplicate.

\section{Critical micelle concentration (CMC) determination}

The CMC of the binary mixture of EPC-SGC (the optimal ratio) in DI water was determined by the pyrene $1: 3$ ratio method. ${ }^{29}$ Samples of MMs of different concentrations ranging from 4\% to $1 \times 10^{-40} \%$ were prepared. Pyrene, dissolved in methanol, was added to EPC-SGC-MMs with different concentrations to obtain a final concentration of $4 \times 10^{-7} \mathrm{~mol} / \mathrm{L}$. The mixtures were balanced for 24 hours in a dark room at room temperature before measurement and plated in 96-well plates for fluorescence measurements (using a Fluoroskan Ascent FL; Thermo Scientific, Waltham, MA). Fluorescence emission spectra of pyrene were obtained using an excitation wavelength of $336 \mathrm{~nm}$, with an emission slit of $5 \mathrm{~nm}$. The intensities, $\mathrm{I}_{1}$ and $\mathrm{I}_{3}$, were measured at the wavelengths corresponding to the first and third vibronic bands located near 372 and $383 \mathrm{~nm}$. All fluorescence measurements were carried out at $25^{\circ} \mathrm{C}$. Experiments were performed in triplicate. The ratio of $\mathrm{I}_{1}$ to $\mathrm{I}_{3}$ for pyrene was $1: 3{ }^{29}$ The pyrene $1: 3$ ratio data were analyzed with Origin 8.5 software (OriginLab Corp, Northampton, MA).

\section{Measurement of particle size}

The particle size and polydispersity index of the formed optimal MMs were measured by dynamic light scattering (Malvern Zetasizer Nano ZS90, Malvern Instruments Ltd, Malvern, UK) at $25^{\circ} \mathrm{C}$. The samples were measured with no dilution.

\section{Transmission electron microscopy}

The morphology of optimum NIM-EPC-SGC-MMs was observed with a field-emission high-resolution transmission electron microscope (TEM) (Tecnai G² F20, FEI, Eindhoven, The Netherlands). The sample was placed on copper grids and stained with $2 \%(\mathrm{w} / \mathrm{v})$ phosphotungstic acid for 30 seconds. After the excess solution was drawn off and the sample was allowed to dry, it was subjected to TEM observation.

\section{Drug solubility, solubilizing efficiency, and drug loading of NIM-EPC-SGC-MMs}

The concentration of the NIM solubilized by optimal EPCSGC-MMs was determined by reverse-phase high-performance liquid chromatography (HPLC) on three parallel measurements.
After dilution with methanol, the NIM-EPC-SGC-MMs were injected into an HPLC system. The analysis was performed on an Agilent 1260 infinity LC system (with a G1329B autosampler, G1312B pump, and G1314B detector; Agilent Technologies, Inc, Santa Clara, CA), equipped with a Kromasil C18 column $(250 \times 4.6 \mathrm{~mm}, 5 \mu \mathrm{m}$; Bohus, Sweden $)$ kept at $40^{\circ} \mathrm{C}$ as the stationary phase. The analysis parameters for NIM were as follows: the mobile phase consisted of a mixture of methanol, acetonitrile, and DI water (35:38:27); the injection volume of the samples and standards was $20 \mu \mathrm{L}$; the flow rate was set at $1.0 \mathrm{~mL} / \mathrm{min}$, and the detection wavelength was $237 \mathrm{~nm}$ by ultraviolet detection.

Solubilizing efficiency and drug loading (DL\%) were calculated by the following equations:

Solubilizing efficiency (\%)

$=($ Weight of NIM in micelles

/Weight of NIM feeding) $\times 100 \%$

DL $\%=($ Weight of NIM in micelles/Weight of feeding EPC and SGC and NIM) $\times 100 \%$

\section{Physical compatibility study with sodium chloride injection, glucose injection,} and sterile water

The NIM-EPC-SGC-MMs and NIM injection were diluted by certain degrees - 10-fold, 100-fold, 500-fold - by sodium chloride injection, glucose injection, and sterile water, respectively. Each sample was placed in a $10 \mathrm{~mL}$ tube and examined for observable small particles and low-level haze $^{30}$ in normal fluorescent light from a Tyndall beam (high-intensity mono-directional light source) using the unaided eye. As a standard, the turbidity of each sample was measured. All of the samples were kept at room temperature and observed at fixed time points $(0,0.5,1,5$ and 10 hours).

\section{In vivo studies in rats}

Ten male Wistar rats $(200 \pm 20 \mathrm{~g})$ were randomly divided into two groups, with five rats in each group. Rats were fasted overnight but supplied with water before the experiment. The NIM injection and NIM-EPC-SGCMMs, with the same mass concentration of NIM, were administered by intravenous injection through the tail vein at a dose of $2 \mathrm{mg} / \mathrm{kg}$.

After administration, blood was collected from the ocular vein at predetermined time points $(5,15,30,45,60,120,240$, 300 minutes $)$. Plasma samples $(0.5 \mathrm{~mL})$ were placed into 
heparinized centrifuge tubes; plasma samples $(100 \mu \mathrm{L})$ were collected immediately following centrifugation at $1296 \times g$ for $10 \mathrm{~min}$ and then stored at $-20^{\circ} \mathrm{C}$ until further use.

\section{Determination of NIM in plasma samples by HPLC}

The concentration of NIM in blood was determined by a reverse-phase HPLC method. ${ }^{31}$ In brief, $100 \mu \mathrm{L}$ of plasma was mixed with $25 \mu \mathrm{L}$ of internal standard (nitrendipine at a concentration of $0.0020 \mathrm{mg} / \mathrm{mL}$ in methanol), followed by vortex mixing for 1 minute. Then, $50 \mu \mathrm{L}$ of $1 \mathrm{~mol} / \mathrm{L} \mathrm{NaOH}$ was added and the mixture was extracted with $0.4 \mathrm{~mL}$ $\mathrm{N}$-hexane-ethyl ether (1:1) twice. After vortex for 1 minute and centrifugation at $12,000 \times g$ for 10 minutes, the supernatant was collected and combined, followed by evaporation under nitrogen at $50^{\circ} \mathrm{C}$. The residue was dissolved into $100 \mu \mathrm{L}$ mobile phase after vortexing for 30 seconds and centrifuged at $12,000 \times g$ for 10 minutes sequentially; $50 \mu \mathrm{L}$ of the resultant supernatant was subjected to HPLC analysis. A reverse-phase column $(\mathrm{C} 18,250 \times 4.6 \mathrm{~mm}, 5 \mu \mathrm{m})$ was used with a security guard column $(\mathrm{C} 18,10 \times 4 \mathrm{~mm}, 5 \mu \mathrm{m})$, (Phenomenex, Torrance, CA). Under a column temperature of $35^{\circ} \mathrm{C}$, the mobile phase consisted of a mixture of acetonitrile $-0.05 \mathrm{~mol} / \mathrm{L}$ ammonium acetate (60:40). The flow rate was $1.0 \mathrm{~mL} / \mathrm{min}$ and the detection wavelength was $358 \mathrm{~nm}$.

\section{Vascular irritability study in rabbits}

Six male rabbits (weight $2.5-3.0 \mathrm{~kg}$ ) were randomly divided into two groups. One group was injected with NIM-EPCSGC-MMs and sodium chloride injection. The other group was injected with the commercial NIM injection and sodium chloride injection as a control. Each group was injected according to a clinical dosing regimen of $0.4 \mathrm{mg} / \mathrm{kg}$ for 5 days. In brief, animals in the first group were administered an NIM injection of clinical concentration via ear vein infusion on the right and received a sodium chloride injection on the left. The second group was injected with NIM-EPCSGC-MMs of clinical concentration via vein infusion on the right ear and received a sodium chloride injection on the left. Visual observations of the injection site and surrounding tissue were made and recorded as pathological samples at intervals until 48 hours; at that time, each group was sacrificed. After being fixed in $10 \% \mathrm{v} / \mathrm{v}$ formaldehyde in physiological saline for 48 hours, the obtained samples were serially dehydrated by different concentrations of ethanol (100\%, 95\%, 85\%, 70\%, 2-5 minutes for each concentration). The dehydrated samples were embedded in paraffin and cut and stained using hematoxylin and eosin. All sections were observed at $\times 100$ magnification with an Axiovert200 (Zeiss, Oberkochen, Germany), and pathological changes were analyzed and evaluated.

\section{Statistical analysis}

The measured plasma concentration data were analyzed using non-linear regression analysis with the use of Drug and Statistics Software (DAS 2.0; Mathematical Pharmacology Professional Committee of China, Shanghai, China); the best compartment model for estimating pharmacokinetic parameters was then determined. After we analyzed the Student's $t$-test results of the two groups, we determined that a $P$ value $<0.05$ would be considered statistically significant.

\section{Results}

\section{Determination of solubilizing efficiency of NIM-EPC-SGC-MMs in different}

\section{formulations}

The influence of the EPC/SGC mass concentration ratio on the solubilizing efficiency of the NIM-EPC-SGC-MMs (NIM concentration was kept at $0.5 \mathrm{mg} / \mathrm{mL}$ ) is shown in Figure 2 . When the EPC/SGC mass concentration ratio decreased from 1:0.7 to 1:1, a significant increase in NIM solubilizing efficiency was observed. However, a further decrease in the EPC/SGC mass concentration ratio (from 1:1 to 0:1) did not improve NIM solubilizing efficiency - remarkably, it reduced the solubilizing efficiency. The EPC/SGC concentration ratio of 1:1 was used for the subsequent studies, as the solubilizing efficiency under such conditions could be up to $96.40 \%$.

When the mass concentration of NIM in water was set at $0.5 \mathrm{mg} / \mathrm{mL}$, the influence of the total concentration of SGC and EPC on NIM solubilizing efficiency was also

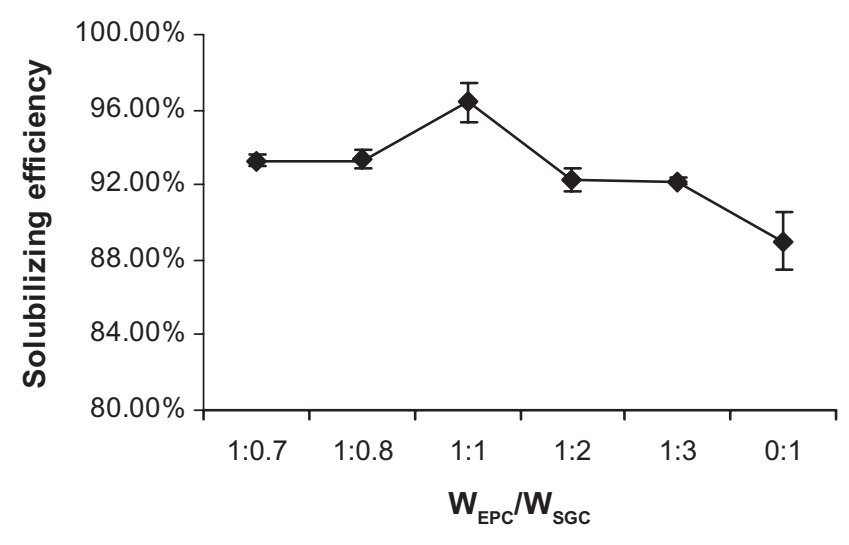

Figure 2 Influence of the mass concentration ratio of EPC and SGC on NIM solubilizing efficiency at $25^{\circ} \mathrm{C}(\mathrm{n}=3)$.

Abbreviations: EPC, egg phosphatidylcholine; SGC, sodium glycocholate; NIM, nimodipine. 


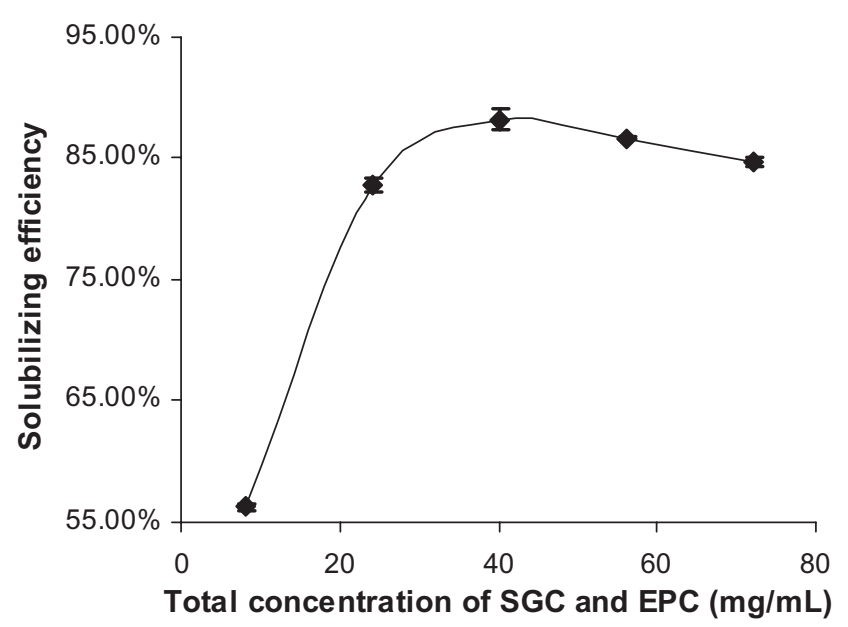

Figure 3 Influence of the total concentration of SGC and EPC on NIM solubilizing efficiency at $25^{\circ} \mathrm{C}(n=3)$.

Abbreviations: EPC, egg phosphatidylcholine; SGC, sodium glycocholate; NIM, nimodipine.

investigated. As shown in Figure 3, NIM solubilizing efficiency was improved by increasing the total concentration of SGC and EPC in the MM system. The solubilizing efficiency did not further improve when the total concentration of SGC and EPC was higher than $40 \mathrm{mg} / \mathrm{mL}$.

The effect of NIM concentration on the stability of the NIM-EPC-SGC-MMs is shown in Table 1. The results showed that NIM concentration could affect the stability of the NIM-EPC-SGC-MMs. The limiting concentration of NIM in this optimized EPC-SGC-MM system was $0.5 \mathrm{mg} / \mathrm{mL}$, since further increases in the concentration of NIM resulted in the instability of the NIM-EPC-SGC-MMs, as indicated by the separation of NIM from the formulation.

The influence of $\mathrm{pH}$ value on the solubilizing efficiency of NIM in EPC-SGC-MMs is shown in Figure 4. An increase in $\mathrm{pH}$ value of the EPC-SGC-MM solvent could dramatically enhance the solubilizing efficiency of NIM when the $\mathrm{pH}$ value was between 2 and 6 . With $\mathrm{pH}$ values higher than 6 , the solubilizing efficiency of NIM showed

Table I Influence of NIM concentration on the stability of NIMEPC-SGC-MMs

\begin{tabular}{lllllll}
\hline NIM (mg/mL) & EPC + SGC (mg) & \multicolumn{7}{l}{ Time (h) } \\
\cline { 3 - 7 } & & $\mathbf{0}$ & $\mathrm{I}$ & $\mathbf{2 4}$ & $\mathbf{4 8}$ & $\mathbf{7 2}$ \\
\hline 0.5 & 40 & $\mathrm{Y}$ & $\mathrm{Y}$ & $\mathrm{Y}$ & $\mathrm{Y}$ & $\mathrm{Y}$ \\
0.8 & 40 & $\mathrm{Y}$ & $\mathrm{Y}$ & $\mathrm{Y}$ & $\mathrm{Y}$ & $\mathrm{C}$ \\
$\mathrm{I}$ & 40 & $\mathrm{Y}$ & $\mathrm{Y}$ & $\mathrm{Y}$ & $\mathrm{C}$ & $\mathrm{C}$ \\
$\mathrm{I} .6$ & 40 & $\mathrm{C}$ & $\mathrm{C}$ & $\mathrm{C}$ & $\mathrm{C}$ & $\mathrm{C}$ \\
2 & 40 & $\mathrm{C}$ & $\mathrm{C}$ & $\mathrm{C}$ & $\mathrm{C}$ & $\mathrm{C}$ \\
\hline
\end{tabular}

Notes: $Y=$ settled solution; $C=$ NIM separating out; $n=3$.

Abbreviations: NIM, nimodipine; EPC, egg phosphatidylcholine; SGC, sodium glycocholate; NIM-EPC-SGC-MMs, nimodipine-egg phosphatidylcholine-sodium glycocholate-mixed micelles.

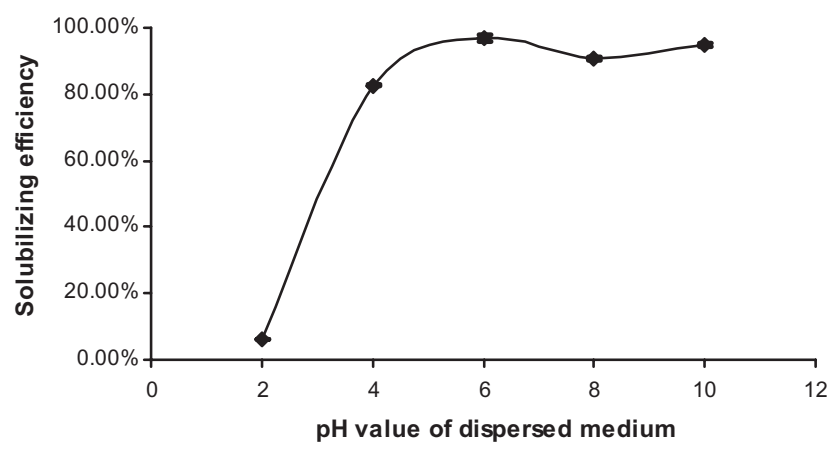

Figure 4 Influence of $\mathrm{pH}$ value of dispersed medium on NIM solubilizing efficiency at $25^{\circ} \mathrm{C}(\mathrm{n}=3)$.

Abbreviation: NIM, nimodipine.

a slight fluctuation. Nevertheless, phospholipids in neutral $\mathrm{pH}$ (6.5) have the greatest stability. ${ }^{32} \mathrm{~A} \mathrm{pH}$ of 7.4 is relevant to physiology. When $\mathrm{pH}$ values were about 7.4, the solubilizing efficiency could be up to $96.40 \%$. Hence, $\mathrm{pH}$ was settled at approximately 7.4 .

The effect of ionic strength on the solubilizing efficiency of NIM-EPC-SGC-MMs was not studied because physiological saline was the only dispersion solution.

\section{CMC determination}

With Origin 8.5 software, pyrene 1:3 ratio plots could be adequately described by the Boltzmann-type approach ${ }^{29,33}$ (Figure 5), which is shown by the following equation:

$$
\mathrm{y}=\frac{\mathrm{A}_{1}-\mathrm{A}_{2}}{1+\mathrm{e}^{\left(\mathrm{x}-x_{0}\right) / \Delta x}}+\mathrm{A}_{2}
$$

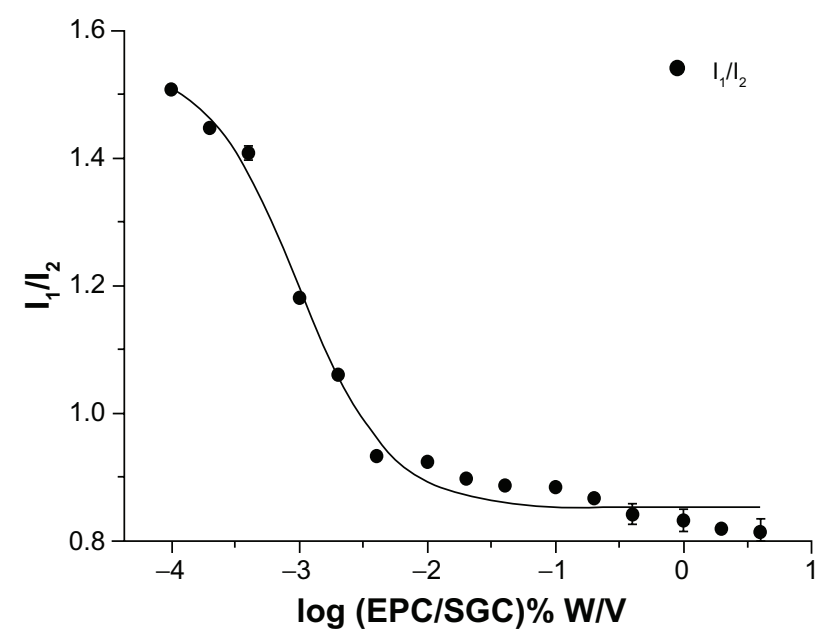

Figure 5 Plot of the fluorescence of pyrene $I_{1} / I_{3}$ intensity ratio versus concentration of EPC/SGC mixed micelles (mass ratio I:I) in distilled water at $25^{\circ} \mathrm{C}(n=3)$.

Abbreviations: EPC, egg phosphatidylcholine; SGC, sodium glycocholate; $I_{1} / I_{3}$, intensity of the third to the first peak ( 372 and $383 \mathrm{~nm}$ ) of pyrene. 
where the variable $y$ is the pyrene 1:3 ratio value, the independent variable $(\chi)$ is the total concentration of surfactant, and $A_{1}$ and $\mathrm{A}_{2}$ correspond to the upper and lower limits of sigmoid, respectively; $\chi_{0}$ is the center of the sigmoid and $\Delta \chi$ is directly related to the independent variable occurence. As shown in Figure 5, the shape of the plots exhibited a typically sigmoidal decrease. For the Boltzmann-type equation, the values for $\mathrm{A}_{1}, \mathrm{~A}_{2}, \chi_{0}$, and $\Delta \chi$ were $1.55,0.85,-3.02$, and 0.36 , respectively. $R^{2}$ was 0.99 , indicating that the Boltzmann-type model was consistent with the typically sigmoidal decrease of the plot. As the value of $\chi_{0} / \Delta \chi$ was less than $10, \chi_{0}$ should be the $\mathrm{CMC}$ value. ${ }^{29}$ Consequently, the CMC value of EPC/SGC (1:1) was $0.0095 \mathrm{mg} / \mathrm{mL}$.

\section{Drug solubility, solubilizing efficiency, and drug loading of NIM-EPC-SGC-MMs}

The optimized formulation, composed of $1.25 \mathrm{mg}$ NIM, $50 \mathrm{mg} \mathrm{SGC}, 50 \mathrm{mg}$ E80, and $2.5 \mathrm{~mL}$ physiological saline, was further characterized by three separate and parallel measurements. As shown in Table 2, the NIM water solubility, solubilizing efficiency, and drug loading of the optimized NIM-EPC-SGC-MMs were $0.468 \pm 0.009 \mathrm{mg} / \mathrm{mL}$, $93.8 \% \pm 2.2 \%$, and $1.17 \% \pm 0.02 \%$, respectively.

\section{Particle size and morphology}

The mean size of the optimal NIM-EPC-SGC-MMs, based on three separate measurements, was $6.099 \pm 0.048 \mathrm{~nm}$, with a polydispersity index of $0.107 \pm 0.016$ (Figure 6A). In addition, the TEM study showed that the NIM-EPC-SGC-MMs were spherical in shape with particle sizes around $6 \mathrm{~nm}$ (Figure 6B).

\section{Physical compatibility study with sodium chloride injection, glucose injection, and sterile water}

All of the tested infusion solutions were physically compatible with the NIM-EPC-SGC-MMs during the 10-hour observation period at room temperature, with no NIM crystals separating out from the mixed solutions at any degree of dilution. In contrast, crystals appeared only half an hour after the ten-times dilution of commercial NIM injections and immediately after 100- or 500-times dilution.

Table 2 Solubility of NIM-EPC-SGC-MMs

\begin{tabular}{llll}
\hline Sample & $\begin{array}{l}\text { Solubility } \\
(\mathbf{m g} / \mathbf{m L})^{\mathrm{a}}\end{array}$ & $\begin{array}{l}\text { Solubilizing } \\
\text { efficiency }^{\mathbf{a}}\end{array}$ & Drug loading $^{\mathbf{a}}$ \\
\hline NIM-EPC-SGC-MMs & $0.468 \pm 0.009$ & $93.8 \% \pm 2.2 \%$ & $1.17 \% \pm 0.02 \%$ \\
\hline
\end{tabular}

Notes: ${ }^{a}$ Reported as means \pm standard deviation; $n=3$.

Abbreviation: NIM-EPC-SGC-MMs, nimodipine-egg phosphatidylcholine-sodium glycocholate-mixed micelles.

\section{In vivo studies in rats}

The mean plasma concentration-time curves of NIM after intravenous administration of NIM injection and NIM-EPCSGC-MMs to rats (equivalent to $2 \mathrm{mg} / \mathrm{kg}$ of NIM; $\mathrm{n}=5$ ), are shown in Figure 7. The results indicate that the NIM-EPCSGC-MMs and NIM injection share the same trend. For both preparations, in the first 45 minutes, the elimination of NIM from the blood was rapid then became very slow after that. The concentration of NIM was at the minimum of 300 minutes and then could no longer be detected. Table 3 shows the main pharmacokinetic parameters in rats after intravenous injection of NIM injection and NIM-EPC-SGC-MMs (calculated with DAS 2.0 software). The area under the concentration-time curve from time zero to infinity, maximum concentration following administration, mean retention time, and half-life of NIM between the NIM-EPC-SGC-MM group and NIM injection group showed no significant differences $(P>0.05)$, indicating that the two formulations were bioequivalent.

\section{Vascular irritability study in rabbits}

The rabbit-ear vein irritation test was processed to evaluate the irritation of NIM-EPC-SGC-MMs and sodium chloride injection versus NIM injection. Compared with the NIM injection, the NIM-EPC-SGC-MM formulation-treated rabbit group did not show deep respiration. In addition, during infusion of the NIM injection, the rabbits struggled more frequently than those in the NIM-EPC-SGC-MMs and sodium chloride injection group. After 5-days of infusion, the ear vein of the rabbits in the NIM injection group showed vascular congestion and vascular lines were unclear. In this group, edema appeared at the surrounding tissue. In contrast, the ear vein of rabbits injected with NIM-EPC-SGC-MMs and sodium chloride injection only had slight phlebectasia, which disappeared 30 minutes after injection.

We viewed these results in histopathological slices, as shown in Figure 8. The histopathological damage caused by the NIM injection was observed microscopically as vasodilation, hemorrhage and degeneration around the vein wall, degeneration of the vein wall, edema around the vein, inflammatory cell infiltration around the vein, and endothelial cell swelling (Figure 8A). However, the results for NIMEPC-SGC-MMs and sodium chloride injection (Figure 8B and $8 \mathrm{C}$ ) show no histopathological damage.

\section{Discussion}

It has been demonstrated that the PC-BS-MM system has great potential to improve the solubility of poorly watersoluble drugs as a novel delivery vehicle, especially in 
A

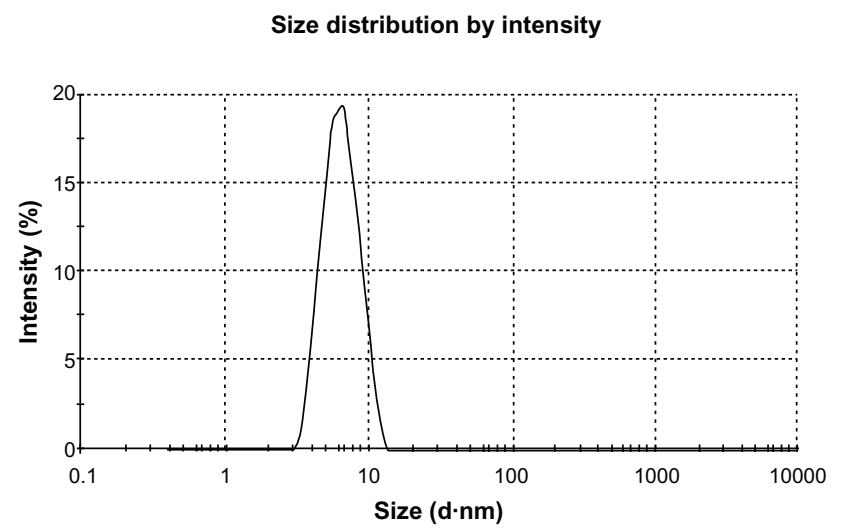

B

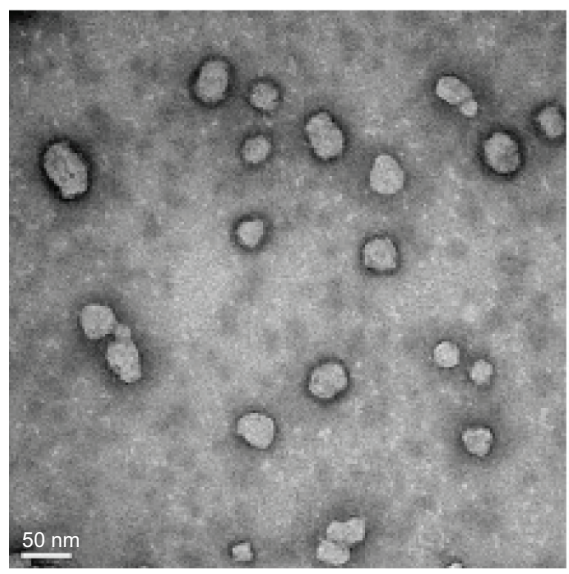

Figure 6 (A) Size and size distribution of the optimal NIM-EPC-SGC-MMs; (B) transmission electron micrograph of NIM-EPC-SGC-MMs (scale bar = 50 nm). Abbreviation: NIM-EPC-SGC-MMs, nimodipine-egg phosphatidylcholine-sodium glycocholate-mixed micelles.

solubilization of some lipophilic drugs. Among different BS, SGC has the highest capacity to solubilize soybean lecithin (SPC), and EPC was solubilized to a higher extent than SPC. ${ }^{15}$ The solubility of NIM in water was $0.468 \pm 0.009 \mathrm{mg} / \mathrm{mL}$ after the formation of optimized NIM-EPC-SGC-MMs. The solubility of NIM in EPCSGC-MMs was significantly improved, about 120 -fold higher than that of free NIM with a water solubility of $3.86 \mu \mathrm{g} / \mathrm{mL},{ }^{10}$ which suggests that the NIM-EPC-SGC-MM system is a promising strategy for improving the solubility of NIM in water.

To optimize the NIM-EPC-SGC-MM preparation, the four main parameters that may affect the solubilization potential of the system were studied: (1) EPC/SGC mass concentration ratio, (2) the total concentration of EPC and SGC, (3) the dose of NIM, and (4) the pH value.

First, the EPC/SGC mass concentration ratio was evaluated and the solubilizing efficiency was employed as the evaluation parameter. The highest solubilizing efficiency of NIM was obtained when the EPC/SGC mass concentration ratio was $1: 1$. The EPC/SGC mass concentration ratio (1:1) was equal to the $\mathrm{EPC}$ mol fraction of 0.6, and $\mathrm{EPC}$ at a maximum mol fraction of 0.6 could be incorporated in SGC to form a clear mixed-micellar solution. ${ }^{15} \mathrm{~A}$ further decrease in the amount of EPC (EPC/SGC mass concentration ratio from 1:1 to $0: 1$ ) did not improve but, remarkably, reduced NIM

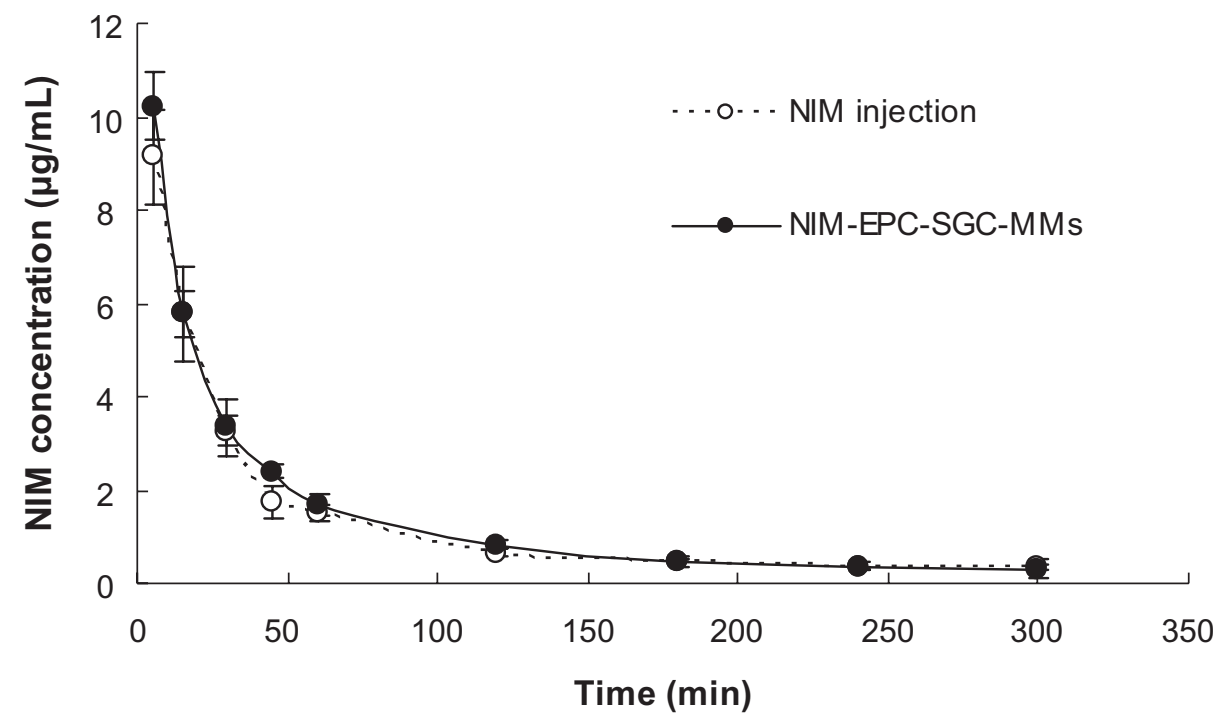

Figure 7 Mean plasma concentration-time curves of NIM after intravenous injection of NIM injection and NIM-EPC-SGC-MMs in rats $(n=5)$. Note: Each point represents mean \pm standard deviation.

Abbreviations: NIM, nimodipine; NIM-EPC-SGC-MMs, nimodipine-egg phosphatidylcholine-sodium glycocholate-mixed micelles. 
Table 3 The main pharmacokinetic parameters of the NIM injection and NIM-EPC-SGC-MMs in rats

\begin{tabular}{lcc}
\hline Parameter & NIM-EPC-SGC-MMs & NIM injection \\
\hline $\mathrm{AUC}_{(0-t)}\left(\mu \mathrm{g} \cdot \mathrm{mL}^{-1} \cdot \mathrm{min}\right)$ & $434.85 \mathrm{I} \pm 30.886$ & $392.903 \pm 34.172$ \\
$\mathrm{AUC}_{(0-\infty)}\left(\mu \mathrm{g} \cdot \mathrm{mL}^{-1} \cdot \mathrm{min}\right)$ & $46 \mathrm{I} .91 \mathrm{I} \pm 26.984$ & $427.848 \pm 23.123$ \\
$\mathrm{MRT}_{(0-\mathrm{t})}(\mathrm{min})$ & $62.722 \pm 5.064$ & $61.999 \pm 9.887$ \\
$\mathrm{MRT}_{(0-\infty)}(\mathrm{min})$ & $83.996 \pm 9.373$ & $95.386 \pm 37.737$ \\
$\mathrm{C}_{\max }(\mu \mathrm{g} / \mathrm{mL})$ & $10.214 \pm 1.136$ & $9.144 \pm 0.797$ \\
$\mathrm{~T}_{1 / 2 z}(\mathrm{~min})$ & $84.312 \pm 6.655$ & $102.394 \pm 46.483$ \\
$\mathrm{CL}_{\mathrm{z}}\left(\mathrm{mL} \cdot \mathrm{min}^{-1} \cdot \mathrm{g}\right)$ & 0.004 & 0.005 \\
\hline
\end{tabular}

Notes: Data reported as means \pm standard deviation; $n=5$.

Abbreviations: AUC, area under the curve; MRT, mean retention time; NIM-EPCSGC-MMs, nimodipine-egg phosphatidylcholine-sodium glycocholate-mixed micelles.
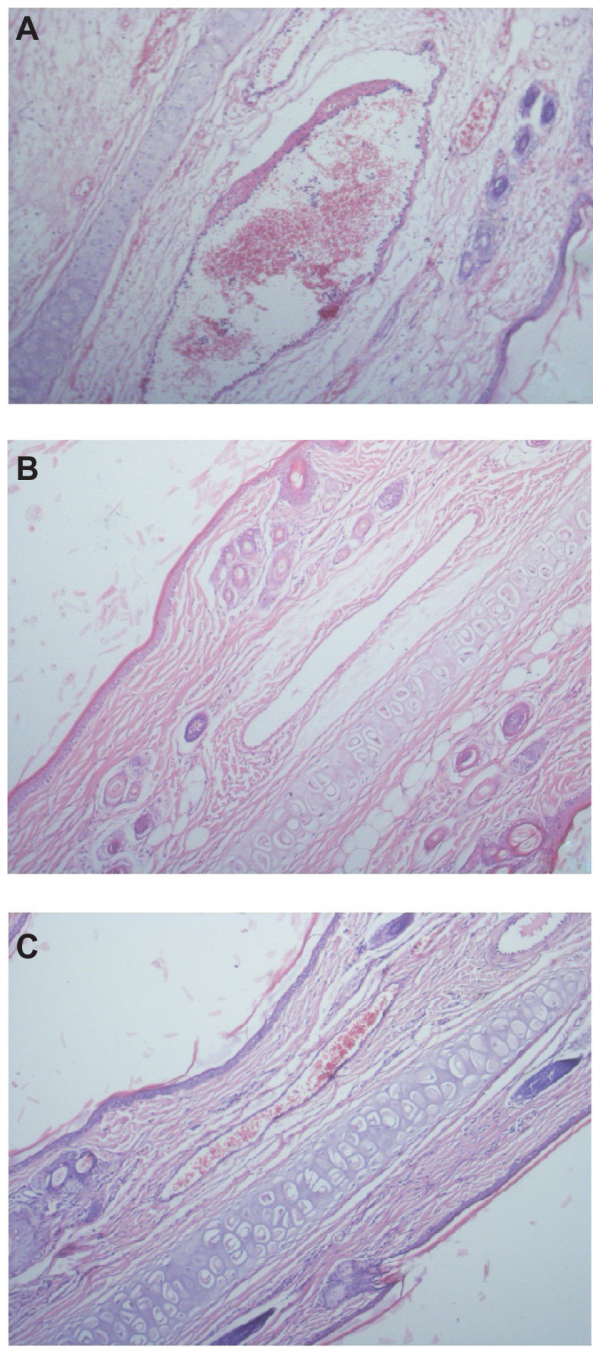

Figure 8 Pathological paraffin sections (hematoxylin-eosin stain) from the ears of rabbits ( $\times 100$ magnification). (A) Ear sample from rabbit given the NIM injection, showing vasodilation, hemorrhage, and degeneration around and of the vein wall as well as edema around the vein, inflammatory cell infiltration around the vein, and endothelial cell swelling. (B) Ear sample from rabbit given NIM-EPC-SGC-MMs. (C) Ear sample from rabbit given sodium chloride injection. The phenomenon shown in (A) was not observed in (B) or (C).

Abbreviations: NIM, nimodipine; NIM-EPC-SGC-MMs, nimodipine-egg phosphatidylcholine-sodium glycocholate-mixed micelles. solubilizing efficiency, indicating that the addition of EPC greatly enhanced the capacity of EPC-SGC-MMs to solubilize NIM. This could be correlated with the ability of the formed MMs to reduce surface tension. ${ }^{17}$ In addition, when the ratio changed from 1:0.7 to 1:1, a significant increase in NIM solubilizing efficiency was observed. It was reported that an increase in the EPC concentration resulted in a higher viscosity and less partition of the drug into the mixed-micellar space. ${ }^{19}$

Regarding the effect of the total concentration of SGC and EPC on the solubility of NIM, the results showed that the optimum total concentration for the NIM-EPC-SGC-MMs was $40 \mathrm{mg} / \mathrm{mL}$. According to the theory of micelle formation, PC-BS-MM formation is associated with the CMC, spontaneous curvature, and chemical structure of different BS and EPC. ${ }^{15,34}$ With an increase in the total concentration of SGC and EPC, the amount of single micelles increased and resulted in an improvement of the solubility of NIM. However, when the NIM within the formulation was all solubilized, the solubilizing efficiency did not improve further when more SGC and EPC were added. In addition, the relatively high solubilizing efficiency achieved by a lower total concentration of SGC and EPC could avoid the administration of redundant excipients.

The formation of drug-loaded micelles is significantly related to the properties of the drug, such as polarity; polarization degree; chain length; chain branching; and molecular size, shape, and structure. ${ }^{15,35}$ As shown by systematic research, ${ }^{15,36}$ the structure of PC-BS-MMs is widely accepted as a "mixed disk model." ${ }^{37}$ In this model, the MM consists of a disk-like core in which BS and phospholipids interact, and BS surround its perimeter. ${ }^{35}$ Drugs are generally inserted into the micelle hydrophobic core or peripheral hydrophilic micellar palisade layer. Like benzodiazepines, ${ }^{15}$ the planar aromatic ring of NIM contributes to the insertion of drug molecules into the lipophilic core of micelles. This corresponds to our results showing that NIM solubility improved when the lipophilic component increased with a greater proportion of EPC. The limiting concentration of NIM was $0.5 \mathrm{mg} / \mathrm{mL}$ in this optimized EPC-SGC-MM system. When the concentration of NIM was more than $0.5 \mathrm{mg} / \mathrm{mL}$, the excess NIM would be temporarily stable in the hydrophilic micellar palisade layer; however, it would gradually separate out from the MM system, as a result of the many oxygen and nitrogen atoms in the NIM molecule, which can easily form hydrogen bonds with water. 
Under ionization conditions, BS have a strong solubilization effect. ${ }^{15-18}$ The $\mathrm{pH}$ value of glycocholic acid is $4.4 .{ }^{15}$ When the $\mathrm{pH}$ was below 4.4, glycocholic acid would precipitate. Thus, when the $\mathrm{pH}$ value increased from 2 to 6 , resulting in an increased amount of ionized SGC, the solubilizing efficiency of NIM in the EPC-SGC-MMs was significantly enhanced. Therefore, considering the solubilizing efficiency, the stability of phospholipids, ${ }^{32}$ and physiological relevance, the $\mathrm{pH}$ value was adjusted to 7.4 ; this moderate $\mathrm{pH}$ value scale was applied to the preparation of the NIM-EPC-SGC-MMs.

We later analyzed some characteristics of the optimized formulation of the NIM-EPC-SGC-MMs, such as particle size, morphology, solubility, solubilizing efficiency, and drug loading. The narrow peak of size distribution and the morphological spherical shape indicated that the NIM-EPCSGC-MMs were relatively uniform and monodisperse.

The pyrene 1:3 ratio method has been reported as one of the most popular methods for measuring the CMC values of both pure micelle solutions and MMs. ${ }^{29,33,38,39}$ Pyrene monomer emission spectra are related to vibronic fine structures, whose intensities are heavily dependent on the polarity of the microenvironment. As a hydrophobic molecule with low aqueous solubility $(\sim 0.3 \mu \mathrm{M})$, pyrene was sensitive to a more hydrophobic environment. ${ }^{38}$ While the pyrene was solubilized in micelles, which resulted in the formation of hydrophobic domains, a significant decrease of $\mathrm{I}_{1} / \mathrm{I}_{3}$ ratio was observed. ${ }^{39}$ The $\mathrm{I}_{1} / \mathrm{I}_{3}$ ratio became finally steady with high surfactant concentration, proving that the pyrene was in a totally hydrophobic environment.

A physical compatibility study with sodium chloride injection, glucose injection, and sterile water was performed to evaluate the safety of the NIM-EPC-SGC-MMs for clinical use. When the NIM injection is diluted, NIM crystals usually appear, owing to the decrease of the organic solvent proportion, which is dangerous for patients. Using EPC-SGC-MMs, NIM could be loaded into the micelle hydrophobic core. During dilution of NIM-EPC-SGC-MMs, the stability of the NIM-EPC-SGC-MMs was associated with the CMC. The entrapped NIM would not separate out from the MM system even when it was diluted 500-fold with the three injection solutions tested and the final total concentration of EPC and SGC was $0.8 \mathrm{mg} / \mathrm{mL}$, suggesting that the concentration of the EPC-SGC-MMs was above the CMC in a wide dilution range. This showed that NIM-EPC-SGC-MMs were physically compatible with these injection solutions.

Pharmacokinetic study in vivo was also performed to further understand the characteristics of NIM-EPC-SGC-MMs.
No significant difference was found in the main pharmacokinetic parameters between NIM injection and NIM-EPCSGC-MMs. This may be due to the quick release of NIM from the MM system when administered by intravenous injection into the blood. To the best of our knowledge, the NIM-EPCSGC-MM system was in dynamic equilibrium. Moreover, the plasma protein-binding rate of NIM was 97\%-99\%. Such a high affinity of NIM to plasma protein could extract NIM from NIM-EPC-SGC-MMs. As free NIM in the NIM injection was also bound with plasma protein, the two formulations share almost the same pharmaceutical profile.

A vascular irritability study in rabbits was performed to evaluate the safety of the formulations. The NIM injection caused a very serious vascular irritability in the ear vein of rabbits, which is in accordance with the results of clinical application. ${ }^{12}$ In contrast, NIM-EPC-SGC-MMs and sodium chloride injection caused almost no vascular irritability. The most reasonable explanation for the irritability of the NIM injection is the effect of the ethanol. ${ }^{11}$ Moreover, NIM could expand the blood vessel, resulting in increased vascular permeability. ${ }^{40}$ Increased vascular permeability would lead to the leakage of plasma, drugs, and ethanol. For treatment of subarachnoid hemorrhage, patients are given a long-term dosage regimen of about 10 hours duration for a continuous 10 to 14 days. ${ }^{41}$ Clearly, the high percentage of ethanol would cause great pain, leading to noncompliance. ${ }^{11}$ Consequently, ethanol and leak damage could be the main causes of the vascular irritation. NIM-EPC-SGC-MMs and sodium chloride injection would not have the undesired side effects associated with the mixed solvent.

\section{Conclusion}

In this study, NIM-EPC-SGC-MMs were prepared using the coprecipitation method. After optimization of the novel formulation, NIM-EPC-SGC-MMs exhibited suitable particle size and high NIM water solubility. NIM-EPC-SGC-MMs were also physically compatible with sodium chloride injection, glucose injection, and sterile water at room temperature for up to 10 hours. Furthermore, the NIM-EPC-SGC-MMs and NIM injection exhibited almost the same pharmacokinetics. There was nearly no vascular irritation caused by the NIM-EPC-SGC-MMs, solving the main problem of NIM intravenous clinical application. As a result, the NIM-EPCSGC-MM system has been proven an attractive method for the intravenous administration of NIM.

Thus, with remarkable improvement in the water solubility of NIM and injection safety, NIM-EPC-SGC-MMs may 
be a promising replacement for the NIM injection, which contains the organic solvent ethanol. In addition, NIM-EPCSGC-MMs can be prepared by a simple and convenient technology, therefore have the potential to be industrially produced on a large-scale.

\section{Acknowledgments}

This work was funded by the National Basic Research Program of China (973 program, No 2009CB930300) and supported by a grant from the National S and T Major Project of China (Grant No 2011ZX09501-001-01). The authors thank Rasa Hamilton (H Lee Moffitt Cancer Center, FL), Jianfeng Han, Qiang Peng, and Sanjun Shi for editorial assistance.

\section{Disclosure}

The authors report no conflicts of interest in this work.

\section{References}

1. Langley MS, Sorkin EM. Nimodipine. A review of its pharmacodynamic and pharmacokinetic properties, and therapeutic potential in cerebrovascular disease. Drugs. 1989;37(5):669-699.

2. Barmpalexis P, Kanaze FI, Kachrimanis K, Georgarakis E. Artificial neural networks in the optimization of a nimodipine controlled release tablet formulation. Eur J Pharm Biopharm. 2010;74(2):316-323.

3. Scriabine A, Schuurman T, Traber J. Pharmacological basis for the use of nimodipine in central nervous system disorders. FASEB J. 1989;3(7): 1799-1806.

4. Hennerici M, Krämer G, North PM, Schmitz H, Tettenborn D. Nimodipine in the treatment of acute MCA ischemic stroke I. Cerebrovasc Dis. 1994;4(3):189-193.

5. Mohr JP, Orgogozo JM, Harrison MJ, et al. Meta analysis of oral nimodipine trials in acute ischemic stroke. Cerebrovasc Dis. 1994;4(3): 197-203.

6. Bekker A, Haile M, Li YS, et al. Nimodipine prevents memory impairment caused by nitroglycerin-induced hypotension in adult mice. Anesth Analg. 2009;109(6):1943-1948.

7. Deshaies EM, Boulos AS, Drazin D, Popp AJ. Evidence-based pharmacotherapy for cerebral vasospasm. Neurol Res. 2009;31(6):615-620.

8. Tomassoni D, Lanari A, Silvestrelli G, Traini E, Amenta F. Nimodipine and its use in cerebrovascular disease: evidence from recent preclinical and controlled clinical studies. Clin Exp Hypertens. 2008; 30(8):744-766.

9. Soliman GM, Sharma R, Choi AO, Varshney SK, et al. Tailoring the efficacy of nimodipine drug delivery using nanocarriers based on $\mathrm{A}_{2} \mathrm{~B}$ miktoarm star polymers. Biomaterials. 2010;31(32):8382-8392.

10. Sun Y, Rui Y, Wenliang Z, Tang X. Nimodipine semi-solid capsules containing solid dispersion for improving dissolution. Int J Pharm. 2008;359(1-2):144-149.

11. Xiong R, Lu W, Li J, Wang P, Xu R, Chen T. Preparation and characterization of intravenously injectable nimodipine nanosuspension. Int J Pharm. 2008;350(1-2):338-343.

12. Liu HM, Zhang P. A study on dexamethasone wet dressing for patients to prevent nimodipine induced phlebitis. Chinese Nursing Research. 2006;20(2):450-451. Chinese.

13. Xiang J, Zhang L, Wang X, Sun X, Wang J, Qi X. Preparation and transdermal diffusion in vitro of nimodipine liposomes. China Pharmacy. 2009;20(1):36-38. Chinese.

14. Alkan-Onyuksel H, Ramakrishnan S, Chai HB, Pezzuto JM. A mixed micellar formulation suitable for the parenteral administration of taxol. Pharm Res. 1994;11(2):206-212.
15. Hammad MA, Müller BW. Increasing drug solubility by means of bile salt-phosphatidylcholine-based mixed micelles. Eur J Pharm Biopharm. 1998;46(3):361-367.

16. Hammad MA, Müller BW. Solubility and stability of tetrazepam in mixed micelles. Eur J Pharm Sci. 1998;7(1):49-55.

17. Hammad MA, Müller BW. Solubility and stability of lorazepam in bile salt/soya phosphatidylcholine-mixed micelles. Drug Dev Ind Pharm. 1999;25(4):409-417.

18. Son K, Alkan-Onyuksel H. Physical stability of teniposide in bile saltegg phosphatidylcholine mixed micelles and liposomes. PDA J Pharm Sci Technol. 1996;50(2):89-93.

19. Duan RL, Sun X, Liu J, Gong T, Zhang ZR. Mixed micelles loaded with silybin-polyene phosphatidylcholine complex improve drug solubility. Acta Pharmacol Sin. 2011;32(1):108-115.

20. Ren CJ, Gong T, Sun X, Zhang ZR, Zhang Y. Alpha-Asarone incorporated in mixed micelles suitable for intravenous administration: formulation, in-vivo distribution and anaphylaxis study. Pharmerzie. 2011;66(11):875-880.

21. Lichtenberg D, Zilberman Y, Greenzaid Y, Zamir S. Structural and kinetic studies on the solubilization of lecithin by sodium deoxycholatet. Biochemistry. 1979;18(16):3517-3525.

22. Rupp C, Steckel H, Müller BW. Solubilization of poorly water-soluble drugs by mixed micelles based on hydrogenated phosphatidylcholine. Int J Pharm. 2010;395(1-2):272-280.

23. Small DM, Penkett SA, Chapman D. Studies on simple and mixed bile salt micelles by nuclear magnetic resonance spectroscopy. Biochim Biophys Acta. 1969;176(1):178-189.

24. Mayer W, Erbe S, Wolf G, Voigt R. Analytik und Stabilität einer pharmazeutisch interessanter 1,4-benzodiazepine. The analysis and stability of various 1,4-benzodiazepines of pharmaceutical interest. Pharmazie. 1974;29(10-11):700-707. German.

25. Claffey WJ, Holzbach RT. Dimorphism in bile salt/lecithin mixed micelles. Biochemistry. 1981;20(2):415-418.

26. Schurtenberger P, Mazer N, Känzig W. Micelle to vesicle transition in aqueous solutions of bile salts and lecithin. J Phys Chem. 1985;89(6): 1042-1049.

27. Steffen H. Prinzip M. Die Gallensäure-Lecithin-Mischmizellen als Trägersystem schwer wasserlösliche Wirkstoffe. [Bile acid-lecithin mixed micelles as a carrier system for poorly water soluble drugs]. Roche Magazine. 1984;20:2-9. German.

28. Teelmann K, Schläppi B, Schüpbach M, Kistler A. Preclinical safety evaluation of intravenously administered mixed micelles. Arzneimittelforschung. 1984;34(11):1517-1523.

29. Aguiar J, Carpena P, Molina-Bolívar JA, Carnero Ruiz C. On the determination of the critical micelle concentration by the pyrene 1:3 ratio method. J Colloid Interface Sci. 2003;258(2): $116-122$

30. Trissel LA, Williams KY, Gilbert DL. Compatibility screening of linezolid injection during simulated Y-site administration with other drugs and infusion solutions. J Am Pharm Assoc (Wash). 2000;40(4): 515-519.

31. Zhang Q, Jiang X, Jiang W, Lu W, Su L, Shi Z. Preparation of nimodipine-loaded microemulsion for intranasal delivery and evaluation on the targeting efficiency to the brain. Int J Pharm. 2004;275(1-2): $85-96$.

32. Grit M, de Smidt JH, Struijke A, Crommelin DJ. Hydrolysis of phosphatidylcholine in aqueous liposome dispersions. Int $J$ Pharm. 1989;50(1):1-6

33. Barbero N, Quagliotto P, Barolo C, Artuso E, Buscaino R, Viscardi G. Characterization of monomeric and gemini cationic amphiphilic molecules by fluorescence intensity and anisotropy. Part 2. Dyes Pigm. 2009;83(3):396-402.

34. Wu TH, Wang ZG. Micellization and phase behavior of biosurfactant bile salts. Process in Chemistry. 2011;23(1):80-89. Chinese.

35. Attwood D, Florence AT. Surfactant Systems: Their Chemistry, Pharmacy and Biology. London and New York, NY: Chapman and Hall; 1983:361-365. 
36. Carey MC, Small DM. The characteristics of mixed micellar solutions with particular reference to bile. Am J Med. 1970;49:590-608.

37. Mazer NA, Benedek GB, Carey MC. Quasielastic light-scattering studies of aqueous biliary lipid systems. Mixed micelle formation in bile salt-lecithin solutions. Biochemistry. 1980;19(4): 601-615.

38. Amiji MM. Pyrene fluorescence study of chitosan association in aqueous solution self-association in aqueous solution. Carbohydr Polym. $1995 ; 26(3): 211-213$.
39. Ménard N, Tsapis N, Poirier C, et al. Drug solubilization and in vitro toxicity evaluation of lipoamino acid surfactants. Int J Pharm. 2012; 423(2):312-320.

40. Lazarewicz JW, Pluta R, Salinska E, Puka M. Beneficial effect of nimodipine on metabolic and functional disturbances in rabbit hippocampus following complete cerebral ischemia. Stroke. 1989;20(1):70-77.

41. Laslo AM, Eastwood JD, Urquhart B, Lee TY, Freeman D. Subcutaneous administration of nimodipine improves bioavailability in rabbits. J Neurosci Methods. 2004;139(2):195-201.
International Journal of Nanomedicine

\section{Publish your work in this journal}

The International Journal of Nanomedicine is an international, peerreviewed journal focusing on the application of nanotechnology in diagnostics, therapeutics, and drug delivery systems throughout the biomedical field. This journal is indexed on PubMed Central, MedLine, CAS, SciSearch ${ }^{\circledR}$, Current Contents ${ }^{\circledR} /$ Clinical Medicine,

\section{Dovepress}

Journal Citation Reports/Science Edition, EMBase, Scopus and the Elsevier Bibliographic databases. The manuscript management system is completely online and includes a very quick and fair peer-review system, which is all easy to use. Visit http://www.dovepress.com/ testimonials.php to read real quotes from published authors.

Submit your manuscript here: http://www.dovepress.com/international-journal-of-nanomedicine-journal 